\title{
A COMPREHENSIVE METHOD FOR IMAGE CONTRAST
}

\section{ENHANCEMENT BASED ON GLOBAL -LOCAL CONTRAST AND LOCAL STANDARD DEVIATION}

\author{
Archana Singh ${ }^{1}$, Neeraj Kumar ${ }^{2}$ \\ ${ }^{1}$ Assistant Professor, Ch. Beeri Singh College of Engg \& Management, Agra, India \\ ${ }^{2}$ Software Developer, Technology9Labs, New Delhi, India
}

\begin{abstract}
Image contrast enhancement is used to increase the detailed visibility of an image by performing some transformation on input image. Global contrast enhancement and local contrast enhancement both are the very popular method for contrast enhancement. But these two methods are also not sufficient in order to enhance an image when image has some constant area and it is not possible to perform any kind of transformation to neighbor hooding pixels of the central pixel. Due to that some information may be lost. This paper proposed a method to remove the divided by zero condition, arises due to the local standard deviation of that constant area in order to enhance an image. This method enhances an image without any loss in detailed visibility of an image.
\end{abstract}

Keywords - contrast enhancement, local contrast enhancement, global contrast enhancement, local standard deviation $* * *$

\section{INTRODUCTION}

The first task of digital image processing is image contrast enhancement. It is done by applying some transformation function for the removal of noise and amplification of the detail that is present in image. Producing image with good contrast and quality is well suitable for biomedical image analysis, fault detection, robotics etc. Contrast enhancement taught to eliminate the problem like unnatural contrast present in the image or image may not reveal all the information present in the captured image. Different methods have already been developed for contrast enhancement [5-6].

Linear stretching and histogram equalization are two widely used methods for global contrast enhancement [12]. These methods are based on the global information of the image. Linear stretching is done by stretching the gray values of input image linearly as shown in fig. 1 . The formal linear stretching adjusts an image's dynamic range and the latter uses the input mapping relation obtained from the integral of the image histogram [3].

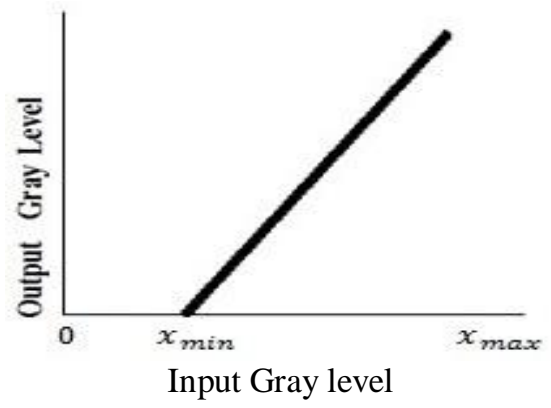

Fig. 1 Linear Transformation
Histogram equalization is one of the most promising techniques for image contrast enhancement. It redistributes the gray level of the image according to the probability distribution of the input gray level [4] as shown in fig.2 and fig.3. This method works well with smaller images or images having different intensity levels. Global histogram equalization and local histogram equalization both comes under the categories of histogram equalization.

Global Histogram Equalization [GHE] is another method for global enhancement. It is very effective and appropriate enhancement method for some images [13].

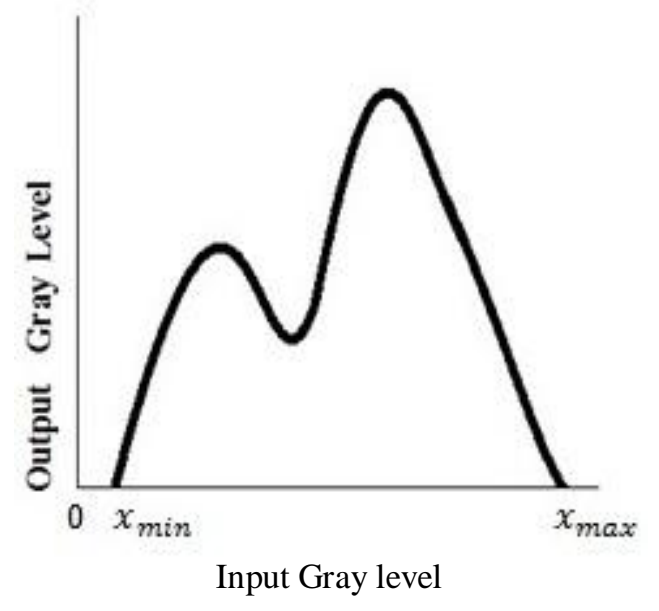

Fig. 2 Histogram Equalization before enhancement 


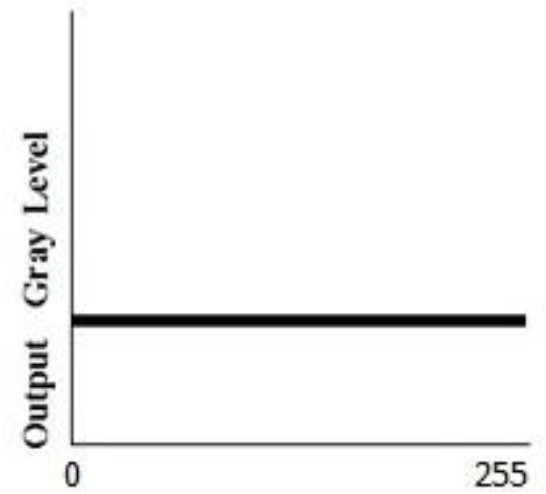

Fig. 3 Histogram Equalization after enhancement

This method has the undesired effect of over emphasizing noise as the gray levels with high frequency rules over the gray levels with low frequency. This method works with the image globally and do not take local details into account. It uses its transformation function to enhance an image based on the histogram input of entire image.

Local histogram equalization is the extension of global histogram equalization [1]. This method is based on block processing. A small sized window is selected from the image for enhancement. This window slides through every pixel of the image in order to enhance that image as shown in fig.4. Only those pixels are considered that fall in this window and enhancement is performed only for the center pixel of that window. Unlike global contrast enhancement this method includes local information of the image.

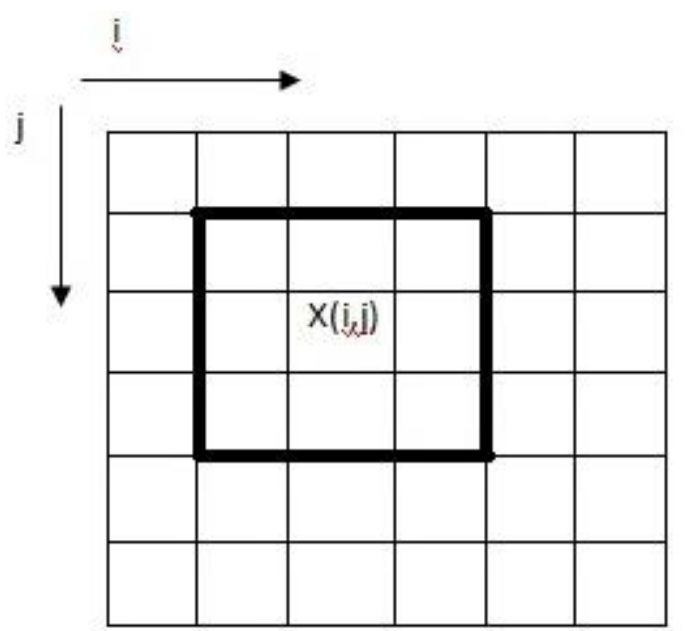

Fig. 4 Local histogram equalization

\section{REVIEW ON LSD BASED METHOD}

Contrast of an image depends upon the local standard deviation [LSD] of the luminance present at the local image area.

Kotkar-Gharde [7] proposed two methods global and local enhancement using Local Feature Enhancement [LFE] and Bidirectional Smooth Histogram Stretching [BSHS] to enhance an input image. LFE is used to search local features of image and BSHS is used to get bidirectional smooth histogram stretching. Then result is combined to get weighted of local and bidirectional smooth histogram stretching and local enhancement on input image.

Marco et. cl. [2] introduced an algorithm based on local contrast enhancement. This algorithm focuses on locally enhancing contrast in under exposed image regions using a background-based approach.

Mukhopadhyay and Chandra [8] proposed a method that was based on multiscale morphology for local enhancement of gray level of input image. This method emphasizes nonlinear enhancement of region that comes into account, extracted from the image based on its shape, size, scale, etc.

Zhen and Wang [14] introduced a piecewise linear function model for emphasizing the luminance contrast at every local area using its LSD. Adaptive contrast enhancement [ACE] is another method for contrast enhancement based on local standard deviation. ACE is the well known technique in medical image processing based on the contrast gain to adjust the high frequency component of images.

Narendra and Fitch [9] introduced a method in that the contrast gain is inversely proportional to the local standard deviation. In this method the transformation function is based on the local gray level information of the input image.

$$
f(i, j)=m_{x}(i, j)+\frac{D}{\sigma_{x}(i, j)}\left[x(i, j)-m_{x}(i, j)\right]
$$

Dah - Chung[3] adapted a new method for ACE in which contrast gain is non linear function of local standard deviation. If enhancement is done with constant gain or inversely proportional to LSD may produced either ringing artifacts or noise over enhancement due to the use of too large contrast gain in areas with high and low activities.

$$
f(i, j)=m_{x}(i, j)+k(i, j)\left[\frac{x(i, j)-m_{x}(i, j)}{\sigma_{x}(i, j)}\right]
$$

Schutte [11] adopted a method that was based on the local and global information of the input image to enhance an image

$$
O=i+\sum_{j=1}^{k} M \frac{C_{j}}{L S D_{j}}\left(i-m_{j}\right)
$$

\section{REMOVAL OF DIVIDED BY ZERO CONDITION OF LSD}

Global contrast enhancement and local contrast enhancement both methods are individually not good for the images having poor local and global contrast. These types of images require such type of transformation function that considers the local and global information of those images. In our previous work [10] we proposed a method that was based on the combination of global and local enhancement. This method requires local as well as global information of an image to perform enhancement for those information that exists in less dynamic range of the image. 


$$
O=I+\frac{M * C_{i}}{\sigma_{i}}\left[m_{i}-m_{i+1}\right]
$$

This method is not suitable for the enhancement of that area which is constant within the image. Local standard deviation of any constant area is always equal to zero. In the above equation 4, LSD is directly proportional to the contrast gain $\mathrm{M}$ and local contrast control $C_{i}$. If LSD of any constant area is equal to 0 then it is not possible to apply any transformation function on neighbor hooding pixel of that central pixel. This LSD can be modified by adding very small value 's' where $\mathbf{s}>\mathbf{0}$ and negligible value too.

$$
O=I+\frac{M * C_{i}}{\sigma_{i} * S}\left[m_{i}-m_{i+1}\right]
$$

Here $\mathbf{O}$ is the pixel's gray level value of enhanced image corresponding to I pixel's gray value of original image. $C_{i}$ is the local contrast control with the range of $[0,1]$. $\mathbf{M}$ is the global contrast gain control with the range of $[0,1.5] . \sigma_{i}$ is the local standard deviation of the window. $m_{i}$ is the pixel's gray scale value present in the original image and $m_{i+1}$ is the subsequent pixel's gray scale value of the original image. This method slides pixel by pixel into the entire image.

\section{EXPERIMENTAL RESULTS}

This section demonstrates the performance of the proposed method. This proposed methods is tested on four gray scale images- Cameraman, Eight, Pout and Tire. Results of the proposed method are compared with the original image and existing method. The validity of the proposed method is also confirmed perceptually. For the quantitative measurement of the contrast enhancement metric Peak Signal to Noise Ratio (PSNR) is used. Table I shows the PSNR values for different images

Table 1 PSNR values for different images

\begin{tabular}{|l|l|l|l|}
\hline Image & Original & $\begin{array}{l}\text { Existing } \\
\text { work }\end{array}$ & $\begin{array}{l}\text { Proposed } \\
\text { work }\end{array}$ \\
\hline a) cameraman & 21.4807 & 21.7654 & 22.1569 \\
\hline b) eight & 18.0114 & 19.0602 & 19.2854 \\
\hline c) tire & 12.1275 & 12.9249 & 13.2014 \\
\hline d) pout & 16.0029 & 17.9826 & 17.9493 \\
\hline
\end{tabular}

From the table and images it has been observed that the proposed method gives better contrast enhancement than the existing work.

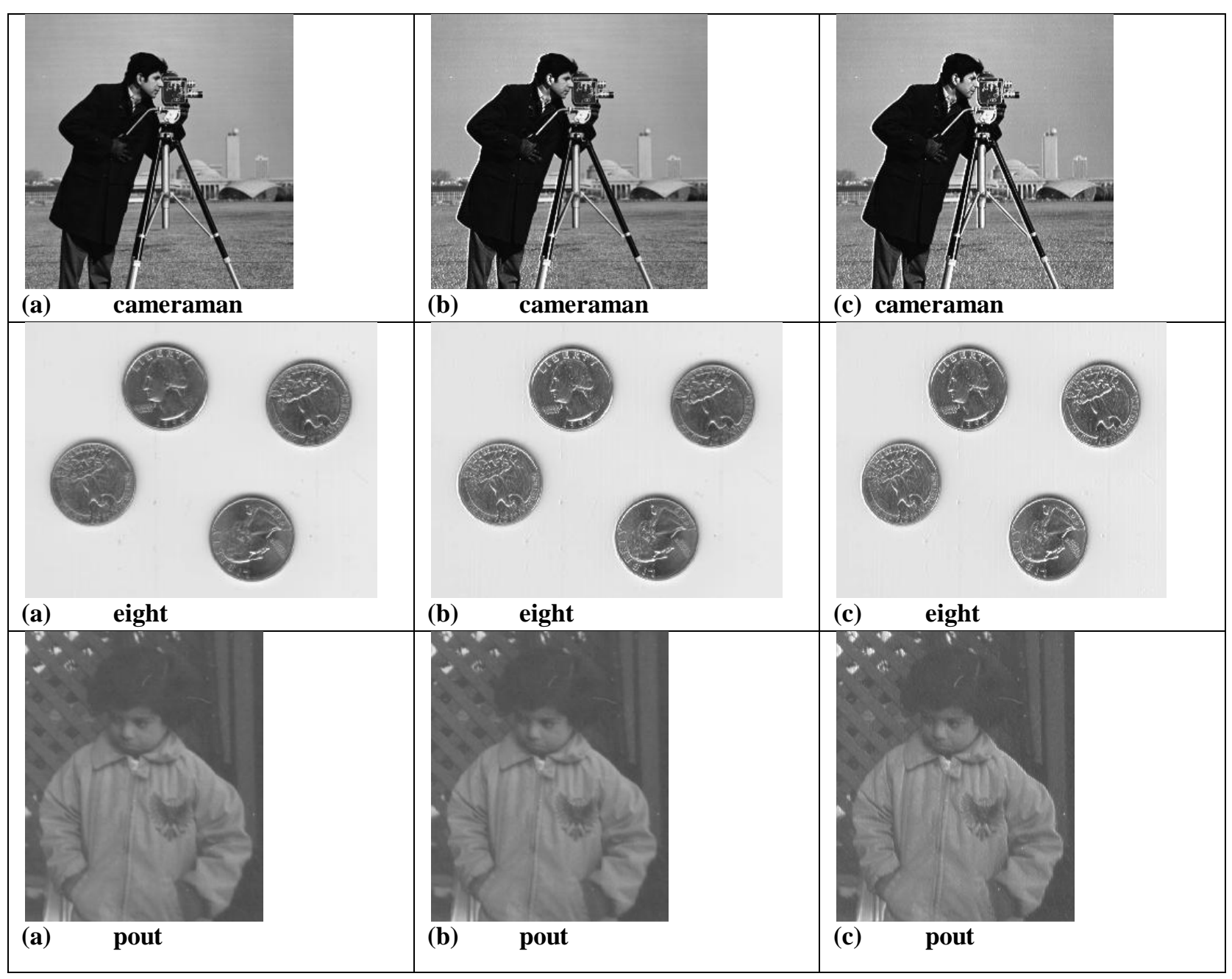




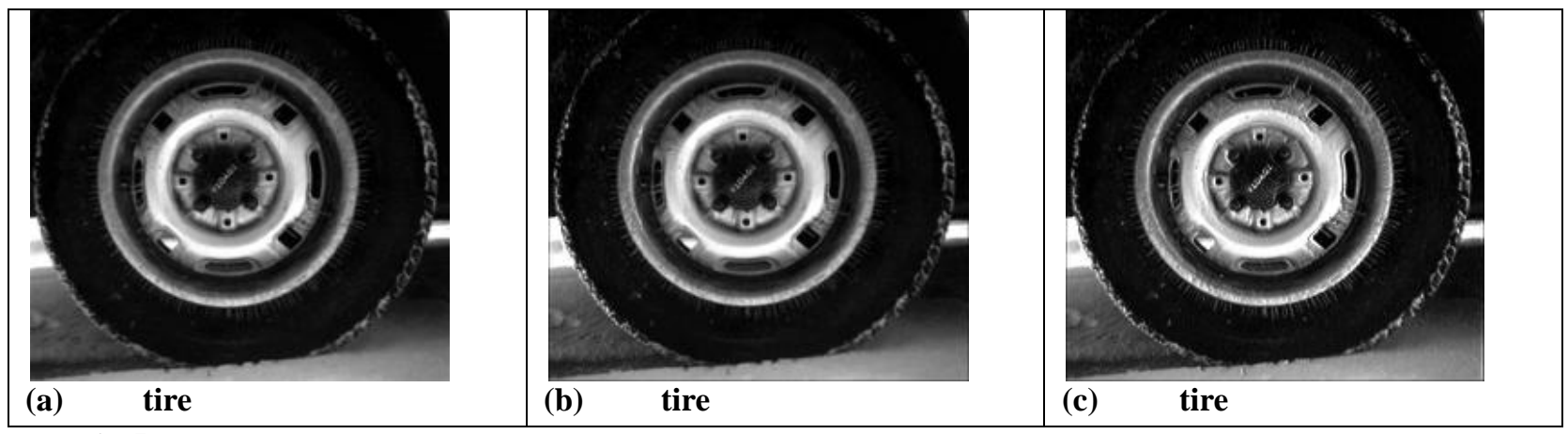

Fig 5 Enhancement results: (a) - Original Image, (b) based on equation no.-3, (c) based on proposed method

\section{CONCLUSIONS}

The basic idea behind this proposed work is to enhance an image using global and local contrast enhancement. This method is based on the local standard deviation. We have removed the divided by zero condition in order to enhance an image. The validity of the contrast of images enhanced by proposed work is confirmed both perceptually and in comparison with other images.

\section{BIOGRAPHIES}

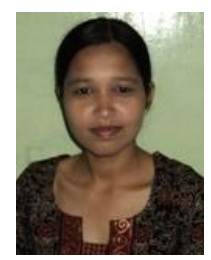

Archana Singh, M.Tech, Digital Image Processing, Ajay Kumar Garg Engg. College Ghaziabad India, Asst. Professor, Department of Computer Science and Engineering, Ch. Beeri singh College of Engg. \& Management, Agra, India.

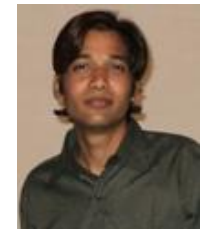

Neeraj Kumar, B.Tech, Computer Science and Engineering, Hindustan College of Science \& Technology Mathura, Software Developer, Software Architecture and Design, Technology9Labs, New Delhi, India.

\section{REFERENCES}

[1]. Archana, Verma, Akhilesh, Goel, Dr. Savita and Kumar, Neeraj. 2013. Gray Level Enhancement to Emphasize Less Dynamic Region within Image Using Genetic Algorithm. In 3rd IEEE International Advances computing Conference IACC-2013, ISBN: 987-1-4673-4528-6.

[2]. Bressan, Marco., Dance, Chistopher R., Poirier, Herve and Arregui, Damian. 2006. Local Contrast Enhancment.

[3]. Chang, D.C. and Wu, W.R. 1988. Image contrast enhancement based on a histogram transformation of local standard deviation. In IEEE Trans. MI, Vol. 17. no. 4, pp. 518-531.

[4]. Gonzales, R. and Winter, P. 1978. Digital Image Processing. Addison Wesley.

[5]. Ibrahim, Haidi and Kang, N.S.P., 2009. Image Sharpening using Sub-Regions Histogram Equalization. IEEE Trans. Consumer Electron. Vol. 55. Issue 2. Pp: 891895

[6]. Jain, K. 1997. Fundamentals of digital image processing. Englewood Cliffs. NJ. Prentice-Hall.
[7]. Kotkar, Vijay A., and Gharde, Sanjay S., 2013. "Review of Various Image Contrast Enhancement Techniques". International Journal of Innovative Research in Science, Engineering and Technology. Vol. 2. Issue-7. ISSN:23198753.

[8]. Mukhopadhyay, S. and Chanda, B. 2000. Local Contrast Enhancement of Grayscale Images using Multiscale Morphology.

[9]. Narendra, P.M. and Fitch, R.C. 1981. Real-time adaptive contrast enhancement," IEEE Trans. PAMI, Vol.3, no. 6 , pp.655-661.

[10]. Singh, Archana and Kumar, Neeraj. 2014. "A GlobalLocal Contrast based Image Enhancement Technique based on Local Standard Deviation". International Journal of Computer Applications. (0975-8887). Vol. 93. No-2.

[11]. Schutte, K. 1997. Multi-Scale Adaptive Gain Control of IR Images. Infrared Technology and Applications XXIII. In Proceedings of SPIE Vol. 3061, pp.906-914.

[12]. Singh, S. Somorjeet, Devi, Dr. H. Mamata, Singh, Th. Tangkeshwar O. and Singh, Imocha. 2012 "A New Easy Method of Enhancement of Low Contrast Image Using Spatial Domain," International Journal of Computer Applications (0975 - 8887) Volume 40- No.1.

[13]. Singh, S.Somorjeet, Singh, Th. Tangkeshwar, Devi, H. Mamata and Sinam,Tejmani. 2012. "Local Contrast Enhancement Using Local Standard Deviation," International Journal of Computer Applications (0975-888). Vol. 47- No.15.

[14]. Zheng, Danian, Wang, Jiaxin and Xiao, Zhenghong. 2005. "Image Enhancement Based on Local Standard Deviation". Journal of Information \& Computational Science 2: 2. 429-437. 